\title{
The density of organized vortices in a turbulent mixing layer
}

\author{
By D. W. MOORE \\ Department of Mathematics, Imperial College, London \\ AND P. G. SAFFMAN \\ Applied Mathematics, California Institute of Technology, Pasadena
}

(Received 16 September 1974)

It is argued on the basis of exact solutions for uniform vortices in straining fields that vortices of finite cross-section in a row will disintegrate if the spacing is too small. The results are applied to the organized vortex structures observed in turbulent mixing layers. An explanation is provided for the disappearance of these structures as they move downstream and it is deduced that the ratio of average spacing to width should be about $3 \cdot 5$, the width being defined by the maximum slope of the mean velocity. It is shown in an appendix that walls have negligible effect.

Brown \& Roshko $(1972,1974)$ have demonstrated experimentally the existence of organized vortex structures in a turbulent mixing layer. The mean spacing $l$ obeys the similarity law

$$
l \propto x,
$$

so that organized vortices must disappear or amalgamate as they are convected downstream, since they are convected with the mean $\bar{U}$ of the velocities on each side of the layer. The annihilation of individual vortices was clearly seen in the experiments.

This phenomenon may be explained qualitatively in terms of the observed tendency of neighbouring vortices to rotate about one another (Winant \& Browand 1974). This rotation is a manifestation of the instability of an infinite row of rectilinear line vortices, of strength $\kappa$ and separation $l$. It is shown in Lamb $(1932, \S 156)$ that for the most unstable disturbance the co-ordinates of the vortices are given by

$$
x_{m}=m l+\epsilon \cos m \pi e^{\lambda t}, \quad y_{m}=\epsilon \cos m \pi e^{\lambda t},
$$

where

$$
\lambda=\kappa \pi / 4 l^{2}
$$

and $\epsilon$ is an arbitrary constant. As the vortices rotate it is assumed that they amalgamate, leading to a new array with larger values of $l$ and $\kappa$. The coalescence of neighbouring vortices leads to a larger vortex. Let $\delta$ denote the diameter of a vortex structure. Then the increase in $\delta$ due to this process is, in this explanation, also taken to account for the growth of the mixing layer with distance $x$ downstream, since $\delta$ can be identified with the width of the mixing layer. 
Support for this view is provided by the numerical experiments of Roberts \& Christiansen (1972), who examined the motion of a pair of vortices of finite cross-section and equal uniform vorticity. The calculation was started with the vortices circular and it was found that if the distance between the centres of the circles was small enough - and about 1.7 of the diameter of the vortices seemed to be the critical distance - the vortices amalgamated. These results are not directly applicable to the infinite array of Winant \& Browand's model, but they are suggestive. Moreover, the orbits of individual vortices in the finite amplitude motion resulting from the initial disturbance (2) are not circular, but are such as to reduce the separation of the vortices by a factor $\div 0.56$ at their closest, which should tend to promote amalgamation on the basis of the ideas to be presented further on.

This explanation is therefore most plausible and appears to describe well many of the observed features. However, difficulties appear on closer examination.

First, Brown \& Roshko did not observe the pairing motion observed by Winant $\&$ Browand. The reason for this observational discrepancy is not known. The distance between individual vortices was comparable to the channel breadth in Brown \& Roshko's experiment, but a detailed calculation of the wall effect on the motion given in (2) shows that it is completely insignificant: even when the channel breadth is equal to the vortex spacing, the growth rate is reduced by only $1 \frac{1}{2} \%$. (See appendix A.)

Second, the picture implies that the vortices amalgamate by a basically inviscid process. For if turbulent diffusion or mixing is invoked during coalescence, it can surely be expected to be operative during the intervening periods, leading to a continuous increase in the size of the vortex structures. This contradicts the notion that increases in the width of the mixing layer are due in the main part to the amalgamation. But if the amalgamation is basically inviscid, the strength of the vorticity in the vortex structures will remain constant because vorticity is conserved in two-dimensional inviscid flow. We are now led in turn to a further contradiction. For,

$$
\kappa / l=\Delta U
$$

where $\Delta U$ is the velocity jump across the mixing layer and $\kappa$ is the strength (i.e. circulation) of the vortex structures. Further,

$$
\kappa=\frac{1}{4} \pi \omega \delta^{2},
$$

where $\omega$ is an average value of the vorticity in the structures, which are taken to be approximately circular. From (4) and (5),

$$
\omega=\frac{4}{\pi} \frac{l \Delta U}{\delta^{2}}
$$

But according to the similarity law, both $l$ and $\delta$ grow linearly with $x$; hence $\omega$ must decrease linearly with $x$.

Thus the assumption that the amalgamation process is inviscid leads to a result which is not consistent with the similarity law. Now if $\omega$ is to decrease the amalgamation must be accompanied by the ingestion of irrotational fluid into the vortex and this requires turbulent mixing and diffusion. 
If turbulent processes act during amalgamation there seems no reason to suppose they do not act continually $f$ and the purpose of the present note is to present an alternative physical mechanism for the disappearance of individual vortices in which continual growth by turbulent entrainment plays an essential role. Nor is pairing essential, though it would act to enhance the process to be described by reducing the separations of the vortices. The new explanation is conceptually simple and moreover leads with the use of reasonable simplifying assumptions to quantitative estimates. It is based on the analytical results of Moore \& Saffman (1971). In particular, we use the result that a steady uniform rectilinear vortex of elliptical cross-section can exist in a uniform irrotational straining field only if

$$
e<0 \cdot 15 \omega
$$

where $\omega$ is the strength of the vorticity and $e$ is the maximum rate of extension. When condition (7) is satisfied, the principal axes of the ellipse are aligned at $45^{\circ}$ to the principal rates of strain, and the ratio $a / b$ of the major and minor axes satisfies the equation

$$
\frac{e}{\omega}=\frac{a}{b}\left(\frac{a}{b}-1\right) /\left(\frac{a^{2}}{b^{2}}+1\right)\left(\frac{a}{b}+1\right)
$$

It can be shown that there are two values of $a / b$ for each value of $e / \omega$ satisfying (7). One value is less than $2 \cdot 9$ and the other greater than $2 \cdot 9$. The more circular shape is stable to two-dimensional disturbances, whereas the other one is unstable. It was not proved that non-elliptical, steady or periodic shapes could not exist when (7) is violated, but numerical experiments, where the vortex core was replaced by point vortices, showed a rapid dispersal of the vorticity when $\omega$ was less than $6 \cdot 7 e$.

The deformation of a vortex in a straining field suggests that the array of circular vortices should be replaced as a simple model of the turbulent mixing layer by an array of elliptical vortices with their major axes parallel to the direction of the flow, where each vortex is deformed by the irrotational straining field of the other vortices. The exact calculation of the shapes is a hard problem, but such refinement is unnecessary for our purposes. We shall simply assume that each vortex has the shape given by a single vortex in a uniform strain, this strain being that at the centre of each vortex when the velocity field of the other vortices is calculated by replacing them by point vortices. A simple calculation (see appendix $\mathrm{A}$ ) then shows that

$$
e=\kappa \pi / 6 l^{2},
$$

and the principal rates of strain are aligned at $45^{\circ}$ to the direction of flow, so that the vortices are aligned parallel to the flow. (The effect of finite channel width is to increase $e$ by a negligibly small amount.) Now,

$$
\kappa=\pi a b \omega .
$$

$\dagger$ We can discern no mechanism by which turbulence (and thus mixing) could be enhanced by the close approach of two like-signed vortices. When the gap between the vortices is much smaller than the radius of curvature the flow can be regarded as parallel, and standard results reveal that it is stable. 


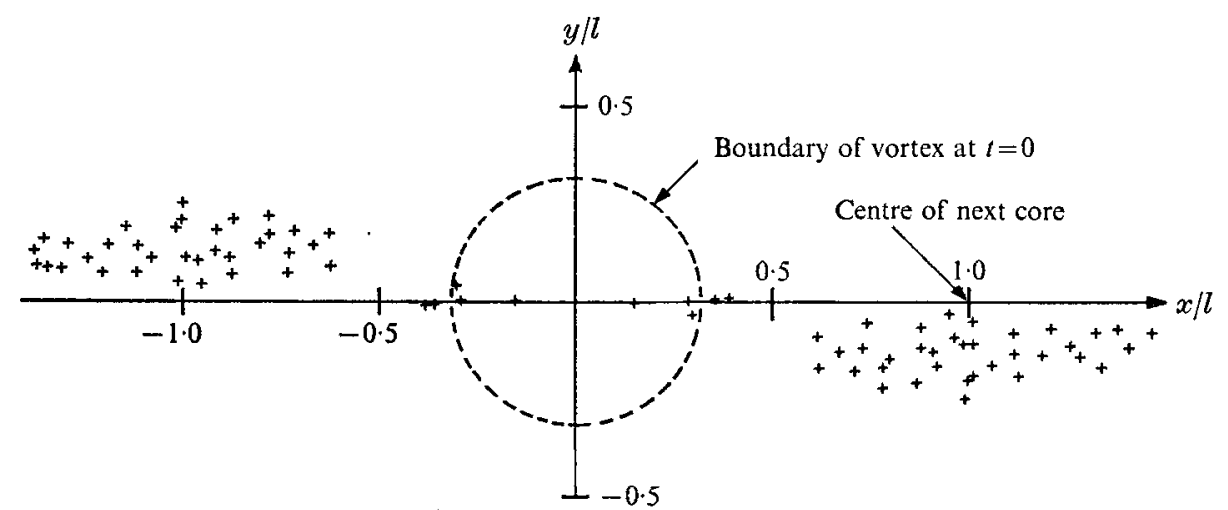

Frgure 1. Position of the 80 line vortices used to represent the vortex core after time $3 \cdot 28 / \lambda$. Only one core of the infinite periodic array is shown. If there is a line vortex at $(x, y)$, there is one at $(x+n l, y)$.

Hence,

$$
e / \omega=\pi^{2} a b / 6 l^{2}
$$

Using the result (7), we deduce that the array can exist as a steady state only if

$$
l>3 \cdot 3(a b)^{\frac{1}{2}} .
$$

This result can be expressed in terms of the width $\delta$ of the array, where $\delta=2 b$. Since $a / b \doteqdot 2.9$ for the critical state, condition (12) is equivalent to

$$
l>2 \cdot 8 \delta \text {. }
$$

Thus our simple analysis gives a lower limit to the distance between the vortices, given their width. If the centres of the vortices are closer than $2 \cdot 8 \delta$, the mutual distortion can be expected to cause the vortices to disintegrate. We note, however, that the distance between the centres is slightly less than the major axis of the ellipses, so that, although the results of the simple model are suggestive, the analysis is being applied outside its strict range of validity. On the other hand, the result of this approach applied to the problem studied by Roberts \& Christiansen agrees reasonably well with theirs (see appendix B).

Moreover, we have tested the argument by examining numerically the motion of an array of initially circular vortices with radius $r$ and separation $l$. According to our theory, the vortices in the array will disintegrate when $r>0.3 l$ and oscillate about an elliptical shape if $r<0.3 l$. Each vortex was replaced by 80 equal-strength line vortices, whose positions at later times were obtained by integration of ordinary differential equations. The line vortices were initially at points of a square lattice inside the circumference of the vortex. Two cases were studied: $r / l=0 \cdot 32$ and $r / l=0 \cdot 26$. In the former case, the vortices are pulled out at an angle close to $45^{\circ}$ to the line joining the centres and are subsequently disrupted. The shape after time $3 \cdot 28 / \lambda$ is shown in figure 1 . Note that reflexional symmetry about the origin was imposed to suppress Lamb instability of the 
array $\cdot \uparrow$ For the latter case, the vortices were observed to oscillate with a period close to $3 / \lambda$; this compares favourably with the theoretical prediction of $2 \cdot 8 / \lambda$ given by equation (3.17) of Moore \& Saffman (1971) (evaluated for an axis ratio of $1 \cdot 7$, which is appropriate for the strain rate at $r / l=0 \cdot 26)$.

It is therefore with fair confidence that we apply the result (13) to the turbulent mixing layer. We envisage the vortex cores as containing turbulent fluid and therefore continuously growing by the entrainment of non-turbulent fluid across their boundaries. Such a process is consistent with the existence of a sharp boundary, and can be represented mathematically as a nonlinear diffusion process. Several of the recent phenomenological models of turbulent shear flow incorporate this idea of nonlinear diffusion to describe the spread of turbulence and predict the propagation of sharp fronts (for example, Saffman 1970). Hence the size of each vortex structure grows continuously with time, or equivalently, distance downstream, and $\delta$ therefore increases. On the other hand, $l$ is constant since the vortices are all convected with the same mean velocity. Thus the condition (13) will eventually be violated and a vortex will be torn apart by the action of its neighbours.

The vortices are not, of course, exactly equal; nor for that matter do they form a spatially homogeneous array as considered in the analysis. There is therefore some physical as well as mathematical extrapolation in applying the analysis to the mixing layer. But the analysis suggests that any vortex which happens to be weaker than its neighbours will be destroyed whenever (13) is violated. When this happens, its vorticity will be ingested by its neighbours. Since the disappearance of a vortex and its amalgamation by a neighbour can be thought of as leading to a doubling of $l$, while $\delta$ is increased only by a factor $2^{\frac{1}{2}}$ (since area is conserved), the process leads to anather stable situation until $\delta$ for the new vortices grows large enough so that (13) is violated, when the process repeats. When a vortex disappears, there is no reason why its vorticity should be shared equally between its neighbours, and if most of the vorticity goes into one of its neighbours, the process may closely resemble the formation of a vortex pair which amalgamate, as was first discussed.

Equation (13) gives a lower bound of $2 \cdot 8 \delta$ for the mean spacing $\bar{l}$ of the vortex structures and a corresponding upper bound of $2 \cdot 9$ for their axis ratio. However, $\delta$ is not a physically well-defined quantity and it is desirable to relate $\bar{l}$ to something that is directly measurable. A suitable length is provided by the maximum slope of the mean velocity gradient through the layer. The mean shear $d \bar{U} / d y$ at a distance $y$ from the centre-line is equal to $\omega$ multiplied by the chance that a point is inside a vortex. A simple calculation shows that the fraction of a line $y=$ constant inside the vortices is

Then

$$
\frac{2 a}{l}\left[1-\frac{4 y^{2}}{\delta^{2}}\right]^{\frac{1}{2}}=f, \text { say. }
$$

$$
d \bar{U} / d y=f \omega .
$$

$\uparrow$ This ensures that the vorticity centroid of each core remains fixed and that pairing does not obscure the dismuption. 
From (4) and (10),

Hence

$$
\omega=l \Delta U / \pi a b .
$$

$$
\frac{d \bar{U}}{d y}=\frac{4}{\pi} \frac{\Delta U}{\delta^{2}}\left(\delta^{2}-4 y^{2}\right)^{\frac{1}{2}}
$$

If we define $\delta_{\omega}$ by

$$
(d \bar{U} / d y)_{\max }=\Delta U / \delta_{\omega}
$$

then

$$
\delta_{\omega}=\frac{1}{4} \pi \delta
$$

and the lower bound on $l$ for a steady array of distorted vortices to exist is $3 \cdot 5 \delta_{\omega}$. Our hypothesis is that individual vortices disappear by disruption and since this process must be continually taking place in order to preserve similarity, this lower bound is achieved. Thus we assert that

$$
\tilde{l}=3 \cdot 5 \delta_{\omega} .
$$

Equation (20) is in reasonable agreement with the measured values reported by Brown \& Roshko (1974), although it is perhaps slightly too large. But the agreement is entirely satisfactory in view of the drastic approximations that have been made and lends support to the belief that the physical mechanism proposed here is responsible for the disappearance of vortices as they are convected downstream.

We do not assert that individual vortices necessarily grow linearly with distance downstream as they increase by turbulent entrainment. In fact, dimensional analysis suggests that the radius of a turbulent vortex should grow like the square root of time. The observed fact that the mean width grows linearly with distance requires that the mean width be determined by the growth due to turbulent diffusion plus the increase in size when a vortex disintegrates and is absorbed by its neighbours.

The present discussion is for a mixing layer in a homogeneous fluid. The qualitative mechanism should hold for mixing between streams of different densities, but the quantitative predictions are questionable.

This work was made possible by a NATO research grant.

\section{Appendix A}

An array of line vortices each of strength $\kappa$ whose centres are the points whose co-ordinates are $(n l, 0)$ relative to fixed rectangular axes produces a flow described by the complex potential

$$
w(z)=-\frac{i \kappa}{2 \pi} \log \left(\sin \frac{\pi z}{l}\right),
$$

where $z$ is $x+i y$. Expanding for small $z$ gives

$$
w=-\frac{i \kappa}{2 \pi}\left[\log z-\frac{\pi^{2} z^{2}}{6 l^{2}}+O\left(\frac{z^{4}}{l^{4}}\right)\right],
$$


so that the perturbing stream function is

$$
\psi=\frac{1}{2}\left(\pi \kappa / 6 l^{2}\right)\left(x^{2}-y^{2}\right)
$$

which is a plane strain of maximum extension rate $\pi \kappa / 6 l^{2}$ as stated in (9).

Next consider the vortices to be placed symmetrically between parallel rigid walls. Suppose that the walls are at $y= \pm h$ with the vortices located as before. The effect of the walls can be accommodated by adding onto (A 1) the complex potential due to an infinite system of image arrays and it is found that

Thus for small $z$

$$
\begin{aligned}
w(z)= & -\frac{i \kappa}{2 \pi} \log \left(\sin \frac{\pi z}{l}\right)-\frac{i \kappa}{2 \pi} \sum_{n=-\infty}^{\infty} \log \left(\sin \frac{\pi}{l}(z-2 n h i)\right) \\
& +\frac{i \kappa}{2 \pi} \sum_{n=-\infty}^{\infty} \log \left(\sin \frac{\pi}{l}(z-\overline{2 n+1} \hbar i)\right) !
\end{aligned}
$$

$$
w(z)=-\frac{i \kappa}{2 \pi}\left[\log z-\frac{\pi^{2} z^{2}}{6 l^{2}}-z^{2} \frac{\pi^{2}}{l^{2}} \frac{(-1)^{n+1}}{\sinh ^{2}(n h \pi / l)}+O\left(\frac{z^{4}}{l^{4}}\right)\right],
$$

so that the perturbing stream function is

$$
\psi=\frac{1}{2} \frac{\kappa \pi}{6 l^{2}}\left[1+6 \sum_{n=1}^{\infty} \frac{(-1)^{n+1}}{\sinh ^{2}(n h \pi / l)}\right]\left(x^{2}-y^{2}\right) .
$$

The infinite series is rapidly convergent unless $h / l \ll 1$ and when $h / l=1$ the effect of the walls is merely to increase the distorting strain by about $4 \frac{1}{2} \%$, an effect completely without significance in the present context.

The effect of the walls on the stability of the array can also be determined. In the mode of maximum growth rate the vortices are displaced to the points

$$
n l+\alpha(t)(-1)^{n}
$$

so that the disturbed array can be regarded as two parallel linear arrays each of spacing $2 l$. This observation enables the velocity potential to be obtained from image arrays and using this potential to evaluate the velocity of one of the vortices leads, on linearizing in the complex disturbance amplitude $\alpha$, to the equation

where

$$
d \alpha^{*} / d t=\left(i \kappa \pi / 8 l^{2}\right)\left\{\left(\alpha-\alpha^{*}\right) R+2 \alpha S-\left(\alpha+\alpha^{*}\right) T\right\},
$$

$$
\left.\begin{array}{l}
R=2 \sum_{n=0}^{\infty} \operatorname{cosech}^{2}\left[\left(n+\frac{1}{2}\right) \frac{\pi h}{l}\right], \\
S=1+2 \sum_{n=1}^{\infty} \operatorname{sech}^{2} \frac{n \pi h}{l}, \\
T=2 \sum_{n=0}^{\infty} \operatorname{sech}^{2}\left[\left(n+\frac{1}{2}\right) \frac{\pi h}{l}\right] .
\end{array}\right\}
$$

Solution of (A 5) shows that the array is always stable, and numerical evaluation of the series shows that the effect of the walls is not practically significant. The results are shown in table 1 , in which $\beta v s$. $l / h$ is given, where $\lambda=\beta \kappa \pi / 4 l^{2}$. 


$\begin{array}{cc}l / h & \beta \\ 0.5 & 1.000 \\ 1.0 & 0.985 \\ 1.5 & 0.884 \\ 2.0 & 0.697 \\ 2.5 & 0.493 \\ 3.0 & 0.324 \\ 3.5 & 0.201 \\ 4.0 & 0.120\end{array}$

Table 1

\section{Appendix B}

We consider the flow examined numerically by Roberts \& Christiansen (1972), which consisted of two circular vortices, of equal strengths $\kappa$ and radii $\alpha$, with centres a distance $l$ apart. With the deformation neglected, the vortices rotate around one another with angular velocity $\Omega=\kappa / \pi l^{2}$. Bring the system to rest by superposing an equal and opposite solid-body rotation. In the new frame of reference, the vorticity inside each vortex is

$$
\omega=\frac{\kappa}{\pi \alpha^{2}}-2 \Omega=\frac{\kappa}{\pi \alpha^{2}}\left(1-\frac{2 \alpha^{2}}{l^{2}}\right) .
$$

Near the centre of one of the vortices, the velocity field induced by the other vortex and the solid-body rotation has stream function ( $x$ axis parallel and $y$ axis perpendicular to the line of centres)

$$
\psi=\frac{1}{2} \Omega\left(x^{2}+y^{2}\right)+\frac{\kappa}{4 \pi l^{2}}\left(x^{2}-y^{2}\right)+\kappa O\left(\frac{a^{4}}{l^{4}}\right)
$$

Applying the analysis of Moore \& Saffman (1971) in the manner used in the body of that paper, equations (2.14) and (2.16), we conclude that the circular vortex is deformed by the velocity field (B 2) into an ellipse of axis ratio $\theta(>1)$, where

$$
\pm \frac{\kappa}{2 \pi l^{2}} \frac{\theta+1}{\theta-1}+\frac{\Omega}{2}=\omega \frac{\theta}{\theta^{2}+1},
$$

the plus sign being taken if the major axis is along the line of centres and the minus sign if it is perpendicular. There are no or two solutions in the former case, and one in the latter. The stability of these shapes to two-dimensional disturbances has been examined, and the only stable shape is the less elongated of the two solutions with major axis parallel to the line of centres, when these exist.

Elementary algebra shows that (B 3), with the positive sign, has real roots $\theta$ greater than one only if

when

$$
2\left(\frac{l^{2}}{\alpha^{2}}-2\right)>\frac{9 \theta_{c}^{2}-2 \theta_{c}+3}{2 \theta_{c}-1}
$$

$$
3 \theta_{c}^{4}-6 \theta_{c}^{3}-2 \theta_{c}-1=0 .
$$


$\theta_{c}$ is approximately $2 \cdot 19$ and is the critical axis ratio. The critical value of $l / 2 \alpha$ is 1.43 . This compares favourably with the numerical value of $1 \cdot 7$ suggested by Roberts \& Christiansen (1972).

\section{REFERENCES}

Brown, G. L. \& Roshko, A. 1972 The effect of density differences on the turbulent mixing layer. Turbulent Shear Flows, AGARD.CP-93, 23-1.

Brown, G. I. \& Roshko, A. 1974 On density effects and large structure in turbulent mixing layers. J. Fluid Mech. 64, 775 .

Lamb, H. 1932 Hydrodynamics. Cambridge University Press.

Moore, D. W. \& Saffman, P. G. 1971 Structure of a line vortex in an imposed strain. In Aircraft Wake Turbulence and its Detection, p. 339. Plenum.

Roberts, K. V. \& Christansen, J. P. 1972 Topies in computational fluid mechanics. Comp. Phys. Comm. (Suppl.) 3, 14.

Saffman, P. G. 1970 A model for inhomogeneous turbulent flow. Proc. Roy. Soc. A 317, 417.

Wrnant, C. D. \& Browand, F. K, 1974 Vortex pairing: the mechanism of turbulent mixing-layer growth at moderate Reynolds number. J. Fluid Mech. 63, 237. 